(C) 2021, American Psychological Association. This paper is not the copy of record and may not exactly replicate the final, authoritative version of the article. Please do not copy or cite without authors' permission. The final article will be available, upon publication, via its DOI: 10.1037/xge0001053

\title{
How (Not) To Test Theory With Data: Illustrations from Walasek, Mullet and Stewart
} (2020)

\author{
Quentin André* \\ Bart de Langhe \\ (Forthcoming, Journal of Experimental Psychology: General)
}

Quentin André (Quentin.andre@ colorado.edu) is assistant professor of marketing at the Leeds School of Business, University of Colorado Boulder. Bart de Langhe (bart.delanghe@esade.edu) is associate professor of marketing at ESADE, Universitat Ramon Llull. Please address all correspondence to Quentin André. The code and files needed to reproduce all the analyses reported in this manuscript are accessible on the OSF repository of the project. 


\begin{abstract}
André and de Langhe (2020) pointed out that Walasek and Stewart (2015) estimated loss aversion on different lotteries in different conditions. Because of this flaw in the experimental design, their results should not be taken as evidence that loss aversion can disappear and reverse, or that decision by sampling is the origin of loss aversion. In their response to André and de Langhe (2020), Walasek, Mullett and Stewart (2020) defend the link between decision by sampling and loss aversion. We take their response as an opportunity to emphasize three guiding principles when testing theory with data: 1) Look for data that are uniquely predicted by the theory, 2) Do not ignore data that contradict the theory, and 3) If an experiment is flawed, fix it. In light of these principles, we do not believe that Walasek, Mullett, and Stewart (2020) provide new insights about the origin and stability of loss aversion.
\end{abstract}




\section{THREE PRINCIPLES WHEN TESTING THEORY WITH DATA}

\section{Look for Data that are Uniquely Predicted by the Theory}

Data that are consistent with a theory may provide confidence that a theory is correct, but that boost in confidence should be modest if the data are also consistent with other plausible and parsimonious mechanisms. To offer an analogy, if your three kids love cookies, how will you know who ate the last cookie?

Walasek, Mullett and Stewart (2021, hereafter WMS) report that the average acceptance rates for common gambles in Walasek and Stewart (2015, hereafter W\&S) differ between conditions. They argue that this pattern "is entirely consistent with the predictions of [Decision by Sampling]." This conclusion is correct. They also argue that "only a model where the wider experience of gains and losses affects the decision to accept the gain/loss pair in a specific question, such as [Decision by Sampling], can do this." This conclusion is not correct.

Suppose participants aim to accept about half of the lotteries they see, favoring those with higher expected values ${ }^{1}$. This "theory" is parsimonious, plausible, and different from decision by sampling. Figure 1 shows the acceptance rates for common gambles in each condition of Study 1 of W\&S that this alternative "theory" would produce. The average acceptance rate is higher when the large majority of other gambles have negative expected values (i.e., in the "G20-L40" condition), and lower when the large majority of other gambles have positive expected values

\footnotetext{
${ }^{1}$ Formally, the decision rule that achieves this result is that the first lottery is accepted with probability $1 / 2$, and the $k^{t h}$ lottery with expected value $E_{k}$ is accepted with probability $\mathrm{p}=\frac{\sum_{i=1}^{k-1} \mathbb{1}_{\left(E_{k}>E_{i}\right)}}{k}$. The code to reproduce this simulation (and all the other figures in the paper) is available on the OSF repository of the project.
} 
(i.e., in the "G40-L20" condition). This simulation shows that the differences in average acceptance rates reported by WMS are not uniquely predicted by decision by sampling, and thus, it does not provide good evidence for this theory.
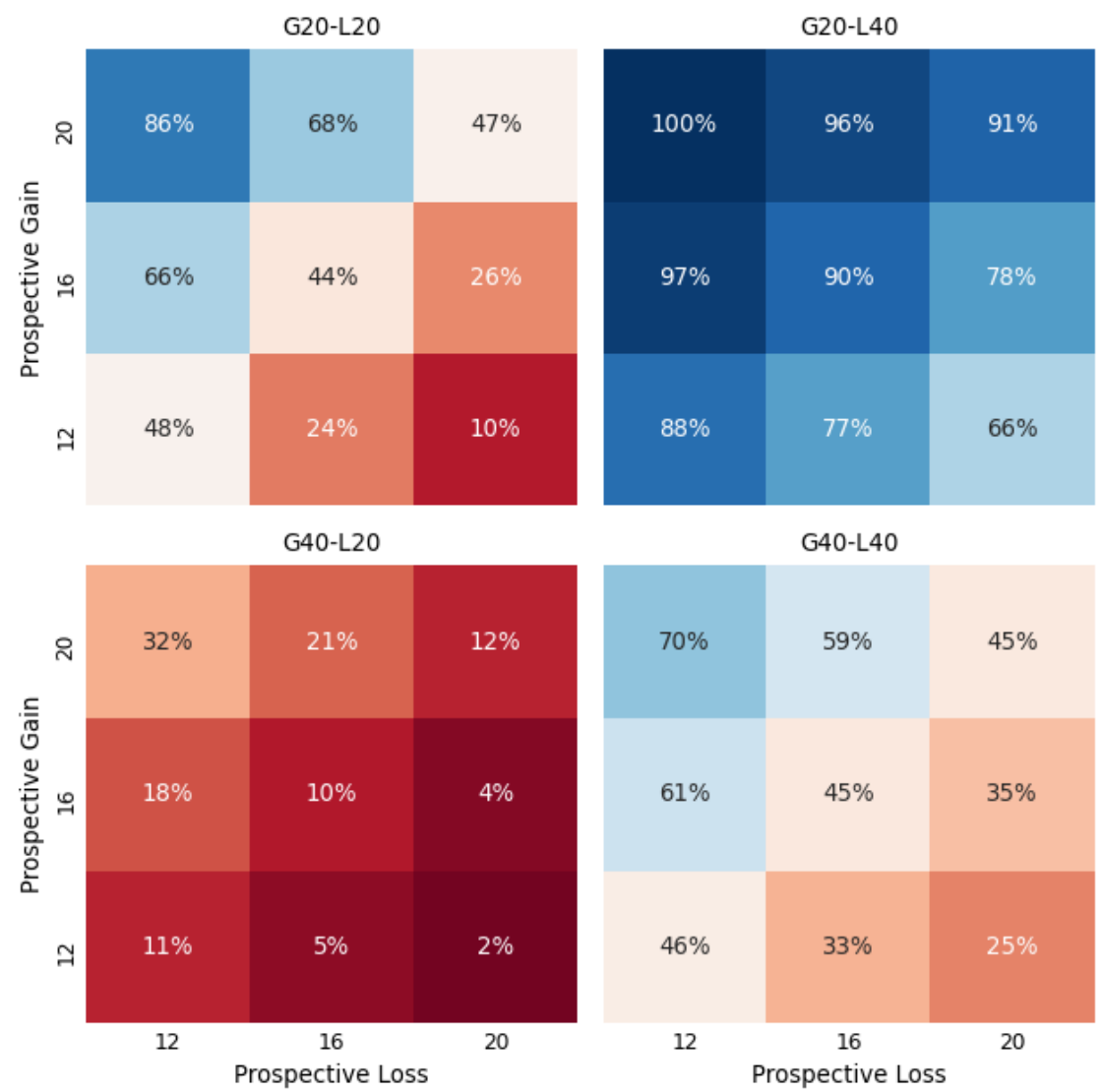

Figure 1: Acceptance rates of common gambles that would be observed if participants aimed to accept about $50 \%$ of the lotteries, favoring those with higher expected values.

Moreover, average acceptance rates for common gambles are not good measures of loss aversion because they are confounded with status quo bias. W\&S had cautioned against using this 
measure as evidence of loss aversion in their introduction ${ }^{2}$, included a $\beta_{\text {bias }}$ parameter in their analytical model to "capture a general tendency to say 'accept' independent of the gain and loss on offer," and insisted in their conclusion that this $\beta_{\text {bias }}$ parameter is what allows them to tease apart differences in loss aversion from differences in status quo bias. Considering these precautions, it is puzzling that WMS use this measure to test the predictions of decision by sampling.

\section{Do Not Ignore Data That Contradict the Theory}

While data that are consistent with a theory do not necessarily provide good evidence for the theory, data that are inconsistent with a theory do provide good evidence against the theory. As Popper argued (1935), one would need infinitely many supporting observations to prove a theory correct, but only one single contradicting observation to falsify a theory. To put it differently, if it walks like a duck, but barks like a dog, it is probably not a duck.

According to decision by sampling, the subjective value of a gain (a loss) is determined by its rank in the set of other gains (losses) in memory. As pointed out by W\&S, this theory predicts that the combination of gains and losses that people have encountered will determine if they are loss averse, loss neutral, or loss seeking. Concretely, in a context of large gains [\$12, \$16, \$20, $\$ 24, \$ 28, \$ 32, \$ 36, \$ 40]$ and small losses [ $\$ 6, \$ 8, \$ 10, \$ 12, \$ 14, \$ 16, \$ 18, \$ 20]$, people should be loss averse. They should be less likely to accept a symmetric gamble with high stakes (e.g., Win $\$ 20 /$ Lose $\$ 20$ with 50\% chance) than a symmetric gamble with low stakes (e.g., Win

\footnotetext{
${ }^{2}$ Citing Gal (2006), they write: "the reluctance to accept a lottery with equal chance of losing and winning the same amount of money should not be interpreted as loss aversion, but instead as a status quo bias."
} 
\$12/Lose \$12 with 50\% chance). This is because, in the context of large gains and small losses, an increase in gains from $\$ 12$ to $\$ 20$ is only "two ranks" better, but an increase in losses from $\$ 12$ to $\$ 20$ is "four ranks" worse. Vice versa, in a context of small gains $[\$ 6, \$ 8, \$ 10, \$ 12, \$ 14$, \$16, \$18, \$20] and large losses [\$12, \$16, \$20, \$24, \$28, \$32, \$36, \$40], people should be loss seeking. They should be more likely to accept a symmetric gamble with high stakes (e.g., Win \$20/Lose \$20 with 50\% chance) than a symmetric gamble with low stakes (e.g., Win \$12/Lose $\$ 12$ with $50 \%$ chance). This is because, in the context of small gains and large losses, an increase in gains from $\$ 12$ to $\$ 20$ is "four ranks" better, but an increase in losses from $\$ 12$ to $\$ 20$ is only "two ranks" worse.

Figure 2 displays the acceptance rates of all common gambles in Study 1 of W\&S. The diagonal cells in each panel represent the acceptance rates for symmetric gambles with small stakes (“Win \$12/Lose \$12," bottom left cell), intermediate stakes (“Win \$16/Lose \$16," middle cell) and large stakes (“Win \$20/Lose \$20," upper right cell). According to decision by sampling, acceptances rates should decrease as a function of stakes in the "G40-L20" condition (people are loss averse), and increase as a function of stakes in the "G20-L40" condition (people are loss seeking). We instead see strikingly similar patterns across conditions, which clearly contradicts decision by sampling ${ }^{3}$.

\footnotetext{
${ }^{3}$ We parenthetically note that the alternative "theory" that we introduced earlier provides a better fit to the data than decision by sampling: A comparison of Figure 1 and Figure 2 shows the same large differences in average acceptance rates between conditions, and the same absence of shifts in the acceptance rates of symmetric gambles with large (vs. small) stakes.
} 

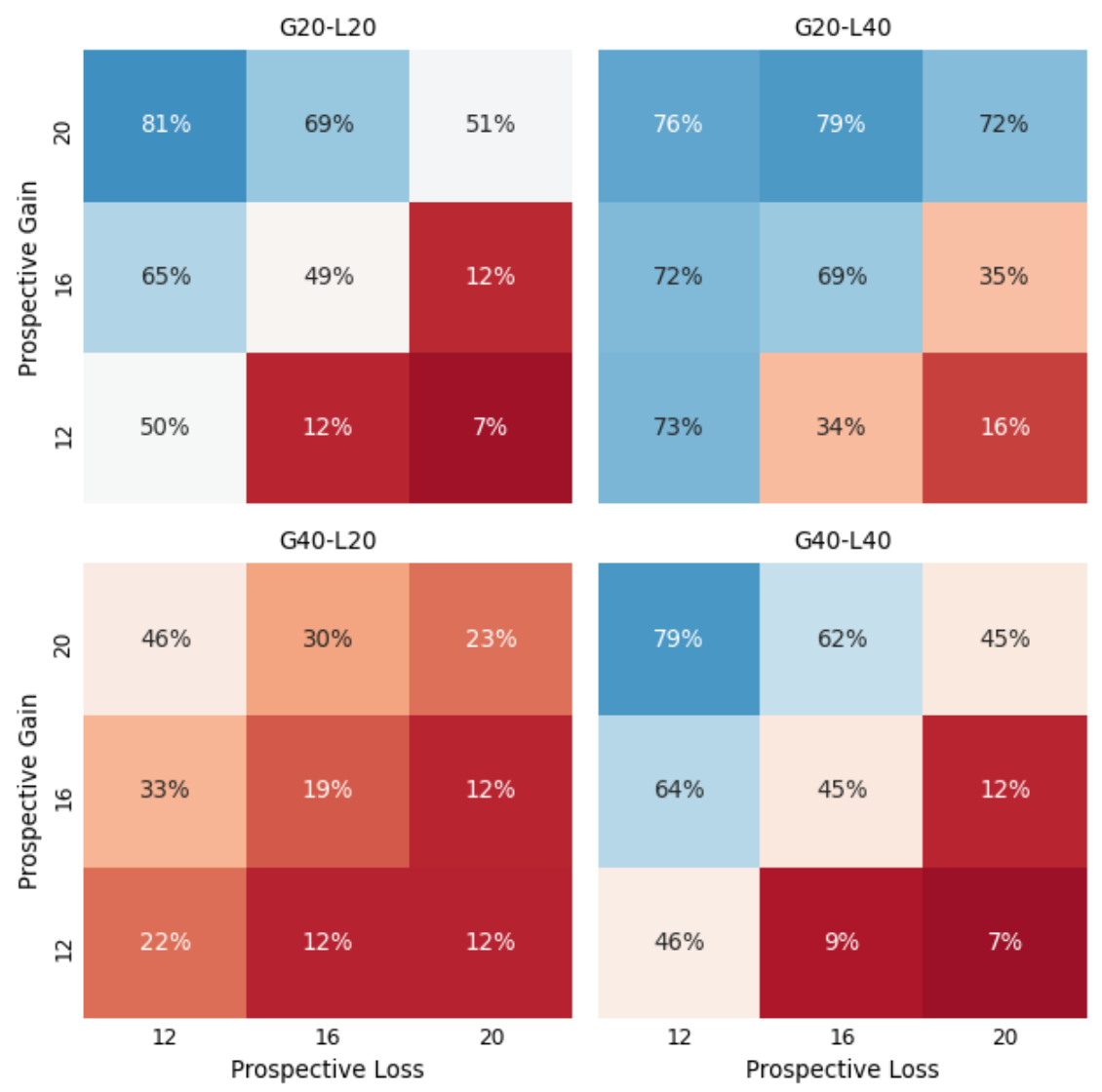

Figure 2: Acceptance rates of common gambles in Study 1 of W\&S.

\section{If an Experiment is Flawed, Fix It}

André and de Langhe (2021, hereafter A\&dL) pointed out that W\&S have estimated loss aversion on different lotteries in different conditions. This is a problem of experimental design, not statistical modeling. Nevertheless, a large part of WMS is devoted to making the argument that individual-level estimates of loss aversion are unstable, and that estimates of loss aversion obtained on one choice set generalize poorly to another choice set. These observations are irrelevant for W\&S and A\&dL. As an analogy, if the engine of your car is broken, fixing the radio should not be your main concern. 
First, the aim of W\&S (and therefore of A\&dL) was not to obtain an individual-level estimate of loss aversion. It was to test the hypothesis that the median level of loss aversion could shift as a function of the distribution of gains and losses that people have encountered. While individual-level estimates of loss aversion may be unstable, the law of large numbers guarantees more stability at the population level. Indeed, the fact that the median estimate for loss aversion obtained from individual choices closely matches the estimate of loss aversion obtained from pooled choices (cf. Appendix of A\&dL) suggests that the individual-level estimates of loss aversion have convergent validity.

Second, a measure does not need to be context-independent to yield useful inferences (Blanton \& Jaccard, 2006). The widespread use of interval scales in experiments shows that theory-testing is rarely about obtaining a point estimate, but rather about making comparisons across conditions. No researcher would for instance expect that participants" "anxiety level" or "reaction time" measured in one context would generalize to another context. Yet, in a valid experimental design, these variables can be used to compare the effectiveness of different stress reduction techniques, or the accessibility of different words in memory.

\section{CONCLUSION}

WMS report that the average acceptance rates for common gambles in W\&S are different between conditions. We have shown that these differences are not uniquely predicted by decision by sampling. Average acceptance rates are also not good measures of loss aversion because they are confounded with status quo bias, a point recognized by W\&S and WMS.

During the review process, we had suggested that a better test of loss aversion is to compare the acceptance rates of symmetric gambles with high (vs. low) stakes across conditions. 
While WMS replied that "this is indeed a neat approach for separating loss aversion from status quo bias," (personal communication, December 2, 2020) they chose not to elaborate on it. We analyzed the complete pattern of acceptance rates and found it is inconsistent with decision by sampling, and inconsistent with the suggestion that loss aversion can disappear or reverse.

WMS agree that the experimental designs in W\&S are flawed. However, they argue that the bigger problem lies in statistical modeling, because it is impossible to obtain stable individual-level estimates of loss aversion. We have argued that this point is irrelevant. If the experimental designs in W\&S had been valid, their estimates of loss aversion would have been informative.

Overall, we view the additional analyses reported in WMS as confounded, incomplete, or irrelevant. We therefore stick to our conclusion that W\&S do not provide evidence that loss aversion can disappear and reverse, nor that decision by sampling is the origin of loss aversion. 


\section{REFERENCES}

André, Q. \& De Langhe, B. (2019, November 11). No Evidence of Loss Aversion Disappearance and Reversal in Walasek and Stewart (2015). Retrieved from https://osf.io/67ng8/

André, Q., \& de Langhe, B. (2021). No Evidence of Loss Aversion Disappearance and Reversal in Walasek and Stewart (2015). Journal of Experimental Psychology: General.

Blanton, H., \& Jaccard, J. (2006). Arbitrary metrics in psychology. American Psychologist, 61(1), 27-41. https://doi.org/10.1037/0003-066X.61.1.27

Popper, K. (1935). The Logic of Scientific Discovery (4th ed.). Routledge.

Walasek, L., Mullett, T. L., \& Stewart, N. (2021). Acceptance of mixed gambles is sensitive to the range of gains and losses experienced, and estimates of lambda $(\lambda)$ are not a reliable measure of loss aversion: Reply to André and de Langhe (2020). Journal of Experimental Psychology: General.

Walasek, L., \& Stewart, N. (2015). How to make loss aversion disappear and reverse: Tests of the decision by sampling origin of loss aversion. Journal of Experimental Psychology. General, 144(1), 7-11. https://doi.org/10.1037/xge0000039 